Investigación

\title{
CALIDAD DE VIDA EN CUIDADORES INFORMALES DE PACIENTES CON ALZHEIMER TRAS IMPLEMENTACIÓN DE PROGRAMA MULTICOMPONENTE
}

\section{QUALITY OF LIFE IN INFORMAL CAREGIVERS OF ALZHEIMER'S PATIENTS AFTER IMPLEMENTATION OF A MULTI-COMPONENT PROGRAM}

\section{Ara Mercedes Cerquera Córdoba}

Psicóloga

Doctor en Ciencias Psicológicas

Escuela de Ciencias Sociales, Universidad Pontificia Bolivariana seccional Bucaramanga, Colombia

Ara.cerquera@upb.edu.co

https://orcid.org/0000-0002-6773-1495

\section{Diana Carolina Tiga Loza}

Enfermera

Doctora en Ciencias

Facultad de Fonoaudiología, Universidad Manuela Beltrán Seccional Bucaramanga, Colombia

diana.tiga@docentes.umb.edu.co

https://orcid.org/0000-0002-0089-2067

\section{William Armando Álvarez Anaya}

Fonoaudiólogo

Magíster en Educación

Facultad de Fonoaudiología Universidad Manuela Beltrán Seccional Bucaramanga, Colombia

fonoaudiologia.bga@umb.edu.co

https://orcid.org/0000-0002-7761-986X

\section{Edwin Dugarte Peña}

Licenciado en Estadística

Magíster en Ciencias-Estadística

Escuela de Ingenierías, Universidad Pontificia Bolivariana seccional Bucaramanga, Colombia

Edwin.dugarte@upb.edu.co

https://orcid.org/0000-0002-0323-932X

\section{Leidy Johanna Plata Osma}

Psicóloga, Magíster en Psicología 
Cerquera Córdoba AM, Tiga Loza DC, Álvarez Anaya WA, Dugarte Peña E, Plata Osma LJ, Jaimes Espíndola LR

Facultad de Psicología, Universidad Pontificia Bolivariana seccional Bucaramanga, Colombia

Leidy.plata@upb.edu.co

https://orcid.org/0000-0002-0644-0459

\section{Lisseth Rocío Jaimes Espíndola}

Fisioterapeuta

Facultad de Fisioterapia, Universidad Manuela Beltrán Seccional Bucaramanga, Colombia. lisseth.jaimes@docentes.umb.edu.co

https://orcid.org/0000-0003-2901-7028

Artículo recibido el 5 de noviembre de 2021. Aceptado en versión corregida el 1 de diciembre de 2021.

\section{RESUMEN}

INTRODUCCIÓN: el cuidado de un paciente dependiente tiene mayores implicaciones si el diagnóstico es la enfermedad de Alzheimer, su calidad de vida se ve afectada de manera considerable en los aspectos relacionados con la salud. OBJETIVO: evaluar el efecto de una intervención multicomponente y transdisciplinar en la calidad de vida relacionada con la salud en cuidadores informales de pacientes con Trastorno Neurocognitivo Mayor (TNC) tipo Alzheimer. MÉTODOS: participaron 50 cuidadores informales de pacientes con Alzheimer, empleando el cuestionario SF-36, mediante un ensayo aleatorizado con tres grupos de comparación y con evaluación de la calidad al inicio, y a los 5 y 10 meses. Para encontrar el tamaño del efecto de las intervenciones en la calidad de vida, según los grupos estudiados, se usó un modelo de efectos mixtos con covarianza no estructurada. RESULTADOS: se encontró que quienes recibieron la intervención multicomponente más respiro obtuvieron un mejoramiento de la calidad de vida en todas las dimensiones y componentes excepto en la función física, con efectos sostenidos en seguimiento. CONCLUSIÓN: las intervenciones no farmacológicas multicomponente acompañadas de un relevo del cuidado tienen un efecto positivo en la percepción de calidad de vida en cuidadores.

Palabras clave: Calidad de vida, cuidador informal, Alzheimer, programa multicomponente.

ABSTRACT

INTRODUCTION: The care of a dependent patient has greater implication if the diagnostic is the disease of Alzheimer's, their quality of life is affected considerably in the aspects related to health. OBJECTIVE: to evaluate the effect of a multicomponent and transdisciplinary intervention on health-related quality of life in informal caregivers of patients with Alzheimer-type Major Neurocognitive Disorder (TNC). METHODS: 50 informal caregivers of Alzheimer's patients participated, using the SF-36 questionnaire, 
Calidad de vida en cuidadores informales de pacientes con Alzheimer...

through a randomized trial with three comparison groups and quality assessment at baseline, and at 5 and 10 months. To find the effect size of the quality of life interventions, according to the groups studied, a mixed effects model with unstructured covariance was used. RESULTS: it was found that those who received the multicomponent intervention plus respite obtained an improvement in the quality of life in all dimensions and components except physical function, with sustained effects in follow-up. CONCLUSION: multicomponent non-pharmacological interventions accompanied by a change of care have a positive effect on the perception of quality of life in caregivers.

Keywords: quality of life, informal caregiver, Alzheimer's, multicomponent program.

\section{http://dx.doi.org/10.7764/Horiz_Enferm.32.3.266-282}

\section{INTRODUCCIÓN}

Según la Organización Mundial de la Salud $^{1}$, las enfermedades crónicas de tipo no transmisible se transformaron en un punto de interés mundial, siendo la demencia una de ellas. Reportes de $\mathrm{OMS}^{2}$, muestran que la demencia afecta a 50 millones de personas en el mundo y $60 \%$ de estos viven en países de ingresos bajos y medios. La demencia se clasifica en múltiples y diversas formas, aquella catalogada como la más frecuente, es la derivada de la enfermedad de Alzheimer la cual representa entre $60 \%$ y $70 \%$ de todos los casos de demencia en el ámbito mundial ${ }^{3}$.

En Colombia, entre los años 2009 y 2015, cerca de 250 mil personas fueron atendidas bajo los diagnósticos CIE-10 relacionados con demencia ${ }^{4}$; sin embargo, la prevalencia de la Enfermedad de Alzheimer no tiene datos precisos en el país ${ }^{5}$.

Por otro lado, se ha descrito que la enfermedad de Alzheimer es además una de las que mayor impacto social y económico generan para los sistemas de salud, grupos poblacionales, familiares y los pacientes mismos, pues afecta su funcionalidad y se genera incidencia en la disminución de su calidad de vida ${ }^{6}$. Así mismo, en los cuidadores, esta enfermedad también tiene un nivel de afectación tanto física, psicológica y social, puesto que deben enfrentar los retos que implica el asumir el cuidado en cualquier etapa de la enfermedad, además de contribuir a optimizar la calidad de vida del paciente ${ }^{5-}$ 7 .

En tal aspecto, un papel fundamental lo representan los cuidadores informales, ya que es una experiencia de alta demanda en el desarrollo de actividades fundamentales e instrumentales en la vida diaria de su paciente familiar ${ }^{8}$ que afecta su calidad de vida. El cuidador informal se describe como aquel individuo que dedica la mayor parte del tiempo (en horas), al cuidado de dicho enfermo y que ha realizado las tareas de cuidado durante un mínimo de 6 semanas ${ }^{9}$; sumándose a esto, no recibe remuneración económica de ningún tipo ${ }^{10}$.

Las actividades de cuidado de algún miembro de la familia con deterioro 
cognitivo pueden ser descritas tan extenuantes; además, se considera una labor "extremadamente estresante", generando un alto costo derivado del deterioro físico, psicológico, emocional y espiritual de los cuidadores y sus familias ${ }^{8}$, aspectos claves en la calidad de vida.

Asimismo, el acto del cuidado podría ser un factor que contribuye en la presentación de enfermedades en el cuidador, presentando patologías como la hipertensión con una prevalencia de 12,7\% e insomnio con una prevalencia de $11 \%$, equiparadas al nivel de enfermedad y sumadas a la gravedad del paciente ${ }^{7-11}$.

De esta manera, es evidente que los cuidadores informales también se vean expuestos, de forma directa, a variaciones en su bienestar y calidad de vida ${ }^{6}$. Es así como, la evaluación de esos cambios vinculados al cuidado y el efecto que puedan tener en la calidad de vida debería ser una prioridad en los cuidadores de pacientes con Enfermedad de Alzheimer.

Se ha definido la calidad de vida como la percepción subjetiva del estado de salud actual y la capacidad para desarrollar actividades transcendentales para el individuo $^{12-13}$, por tanto, es necesario realizar una evaluación multidimensional de las situaciones de vida actuales de la persona ${ }^{14}$.

Al respecto, la calidad de vida relacionada con la salud, es un componente que relaciona experiencias subjetivas sobre la salud global ${ }^{15}$, que evaluada en cuidadores permite evidenciar la magnitud y el impacto de la enfermedad sobre la percepción de su bienestar físico, social, mental y espiritual ${ }^{16}$; además de mostrar las consecuencias del cuidado, así como el efecto de intervenciones.
Por tanto, el estudio pretende evaluar el efecto de una intervención multicomponente y transdisciplinar en la calidad de vida relacionada con la salud en cuidadores informales de pacientes con Trastorno Neurocognitivo Mayor (TNC) tipo Alzheimer en el marco del proyecto "Evaluación de impacto de un programa multicomponente con intervención transdisciplinar en la sobrecarga y la calidad de vida relacionada a la salud de cuidadores informales de pacientes con Trastorno Neurocognitivo (TNC) mayor tipo Alzheimer en Bucaramanga y su área metropolitana" financiado por el Ministerio de Ciencia, Tecnología e Innovación (MINCIENCIAS), convocatoria 807-2018 para proyectos de ciencia, tecnología e innovación en salud, por medio de un modelo de efectos mixtos con covarianza no estructurada.

\section{METODOLOGÍA}

\section{Diseño}

El presente análisis se realiza a partir la información obtenida en un estudio tipo ensayo controlado aleatorizado que evaluaba la eficacia de un programa multicomponente con intervención transdisciplinar en la calidad de vida de cuidadores informales de pacientes con Trastorno Neurocognitivo (TNC) mayor tipo Alzheimer en población colombiana, a partir de la comparación de tres grupos en paralelo y una razón de asignación $1: 1: 1^{19}$.

\section{Participantes}

Los criterios de inclusión fueron ser familiar de un paciente con TNC mayor tipo Alzheimer, convivir con el paciente en 
Calidad de vida en cuidadores informales de pacientes con Alzheimer...

el mismo domicilio, ejercer el rol del cuidador mínimo ocho horas diarias durante más de tres meses, y no recibir remuneración económica por desempeñar el rol del cuidador. Por su parte, los criterios de exclusión se basaron en dejar de ejercer el rol de cuidador principal, hospitalización prolongada del paciente antes y durante el estudio, institucionalización del paciente o muerte del cuidador o del paciente con TNC mayor tipo Alzheimer, durante la fase de intervención.

Para la selección de los participantes, se realizó una convocatoria abierta para cuidadores partiendo de un censo de intención de participación, a través de bases de datos existentes de cuidadores informales y el reclutamiento a partir de convocatoria abierta. Las instituciones a las cuales se censó incluían Instituciones prestadoras de servicios de salud (IPS), Entidades Privadas en relación a Salud mental que tenían en sus servicios grupos de Enfermedad de Alzheimer, Centros vida y sus grupos de Adulto mayor, Grupos Religiosos y, el método voz a voz entre la comunidad tras la difusión de un comunicado por escrito o volante impreso. así como sitios que concentraran un mayor número de personas adultos mayores como. Así como a través de medios de comunicación y redes sociales como Facebook y WhatsApp.

\section{Muestra}

Se contactaron a 143 cuidadores, de los cuales, cumplieron criterios de inclusión y aceptaron participar en el estudio 50 que fueron aleatorizados a los tres grupos de la siguiente manera: grupo control $(n=17$ cuidadores), grupo multicomponente más respiro ( $\mathrm{n}=17$ cuidadores) y grupo respiro
( $n=17$ cuidadores). Se destaca que el grupo multicomponente más respiro tuvo una adherencia del $100 \%$ durante todo el tiempo del estudio.

\section{Asignación aleatoria}

Mediante generación de números aleatorios se creó la secuencia aleatoria simple usando el programa Microsoft Excel y ocultada mediante en sobres cerrados. Una vez el cuidador aceptó participar en el estudio, se procedía a conocer a cuál de los tres grupos fue asignado que fueron los siguientes: A) Grupo de intervención multicomponente más respiro, B) Grupo de sólo respiro y C) Grupo control.

\section{Enmascaramiento}

$\mathrm{Ni}$ los participantes, ni los profesionales que realizaron la intervención estuvieron enmascarados, el analista de los datos estuvo cegado con una base de datos en la cual no se identificaron los grupos de comparación.

\section{Instrumentos}

Referente a la evaluación, se decide utilizar el cuestionario de salud SF-36, el cual reporta estándares de calidad del Medical Outcome Trust y de la Red Cooperativa para la Investigación en Resultados de Salud y Servicios Sanitarios (Red IRYSS). Este cuestionario ha sido adaptado a la población colombiana, mostrando propiedades psicométricas adecuadas, pudiendo ser aplicado en personas tanto sanas como enfermas ${ }^{15}$, y ha sido usado en la evaluación del efecto de las intervenciones enfocadas en el cuidador $^{17}$, además, ha mostrado ser 
sensible al cambio frente a intervenciones no farmacológicas para cuidadores ${ }^{18}$.

La evaluación se realizó mediante el cuestionario SF-36 ${ }^{20}$ validado en Colombia ${ }^{15-21}$ el cual consta de 36 preguntas, cubre 8 dimensiones: funcionamiento físico, rol físico, dolor corporal, salud general, vitalidad, funcionamiento social, rol emocional y salud mental del paciente. Para cada dimensión, los ítems son transformados en una escala con un rango de 0 (el peor estado de salud) a 100 (el mejor estado de salud). La medición se realizó en tres momentos: antes de la intervención (línea de base), postintervención 5 meses después y un seguimiento a los 10 meses desde el inicio del estudio. Las aplicaciones fueron llevadas a cabo por el equipo investigador mediante visita domiciliaria.

\section{Programa multicomponente transdis- ciplinar}

Consistió en 8 sesiones de intervención transdisciplinar, desde las áreas de Psicología, Fisioterapia y Fonoaudiología con frecuencia de una vez a la semana y un tiempo estimado de 2 horas, las temáticas a abordar en cada sesión fueron: (1) presentación e introducción al programa, (2) salud mental y bienestar subjetivo, (3) recursos personales, (4) habilidades para el cuidado (5) percepción de apoyo social funcional, (6) relaciones comunicacionales, (7) salud física y (8) red de apoyo profesional.

Dentro del programa se contempló un relevo mediante el cuidado brindado por auxiliares en enfermería que recibieron una capacitación de 8 horas sobre el cuidado a pacientes con enfermedad de Alzheimer, la capacitación fue dada por los profesionales de cada una de las áreas del componente multidisciplinar (psicología, fisioterapia y fonoaudiología), enfatizando en las deficiencias del paciente con Enfermedad de Alzheimer y sus necesidades de cuidado, este relevo se ofrecía en turnos de 8 horas, por 1 día a la semana, durante 8 semanas.

\section{Análisis}

La descripción de las variables sociodemográficas de los pacientes y cuidadores se realizó mediante el cálculo de frecuencias absolutas y porcentajes en el caso de variables cualitativas, las variables continuas, fueron reportadas a través de medias y desviación estándar o medianas y valores mínimos y máximos según test de normalidad de Shapiro Wilk. La búsqueda de diferencias estadísticas en los valores de la calidad de vida según las variables de sociodemográficas y clínicas se realizó a través de las pruebas $\mathrm{T}$ de Student, Kruskal Wallis y Fisher Exacta. Para encontrar el tamaño del efecto de las intervenciones en la calidad de vida, según los grupos estudiados, se usó un modelo de efectos mixtos con covarianza no estructurada. Los análisis fueron realizados en STATA-16 SE S2. $^{22}$

\section{Aspectos éticos}

En relación con la resolución número 8430 de 1993 del Ministerio de Salud Colombiano, por la cual se establecen las normas científicas, técnicas y administrativas para la investigación en salud, esta investigación se consideró de riesgo mínimo, además, se emplearon dos consentimientos informados, uno para cuidadores informales y otro para cuidadores formales contratados para el relevo 
Calidad de vida en cuidadores informales de pacientes con Alzheimer...

profesional. Además, se cumplió con la norma ética establecida de que los sujetos de investigación en grupo control de un ensayo de terapia debieran recibir una intervención de efectividad comprobada ${ }^{23}$. En este sentido, se brindó posterior a la fase de evaluación de seguimiento $\mathrm{y}$, por principio de equidad y beneficencia, al Grupo control, el acceso al componente de "respiro" en un tiempo de 2 meses. Este estudio fue avalado por el comité de ética de la Universidad Pontificia Bolivariana, mediante el aval CE_A_40 del 26 de abril de 2019.

\section{RESULTADOS}

El presente análisis incluyó a 50 cuidadores que completaron como mínimo la evaluación post-intervención a los 5 meses del estudio, durante el periodo de desarrollo de la investigación el cual estuvo comprendido entre los meses de febrero a mayo de 2019. El $76 \%$ de los cuidadores fueron mujeres, con una edad media de 55,1 años y con nivel educativo de secundaria o inferior $(58 \%)^{19}$.

En relación a la calidad de vida se encontró que el grupo de programa multicomponente más respiro) mostraron cambios positivos en todas las dimen- siones del SF-36, lo que evidencia que la ganancias y mejorías en autopercepción, autocuidado y calidad de vida se mantuvieron durante el seguimiento; sin embargo, la dimensión función física no presenta diferencias con significancia estadística en los puntajes para cada momento de la medición ( $\mathrm{p}=0,271 \mathrm{y}$ 0,746); se destaca el rol emocional en el cual se evidencian las mayores diferencias con un incremento de 35,1 puntos en la medición post-test ( $\mathrm{p}=0,003$ ) y de 40,4 hasta los 10 meses de seguimiento $(p=0,001), \quad$ siendo estadísticamente significativos. De igual manera, la dimensión dolor muestra incrementos favorables estadísticamente significativos en 22,5 puntos en la medición post-test y en seguimiento en el seguimiento de 19,8 $(\mathrm{p}<0,001)$. En el Grupo que se utilizó como estrategia el respiro, aunque se presentan cambios positivos en el rol emocional en cada una de las mediciones a través del tiempo, las puntuaciones no son estadísticamente significativas; en el Grupo control no se evidenció un mejoramiento en los puntajes de calidad de vida para ninguna de las dimensiones evaluadas. Lo anterior, se puede evidenciar en la Tabla 1. 
Cerquera Córdoba AM, Tiga Loza DC, Álvarez Anaya WA, Dugarte Peña E, Plata Osma LJ, Jaimes Espíndola LR Tabla 1. Niveles de calidad de vida por dimensión (SF-36) ajustados por modelo de efectos mixtos y diferencias según grupo de intervención y control durante el seguimiento.

\begin{tabular}{|c|c|c|c|c|c|c|c|c|c|}
\hline & \multicolumn{3}{|c|}{$\begin{array}{l}\text { Grupo Multicomponente más } \\
\text { respiro }\end{array}$} & \multicolumn{3}{|c|}{ Grupo respiro } & \multicolumn{3}{|c|}{ Grupo control } \\
\hline & $\begin{array}{l}\text { Media } \\
\text { (IC95\%) }\end{array}$ & $\begin{array}{c}\text { Diferencia } \\
\text { (IC95\%) }\end{array}$ & $\begin{array}{c}\text { Valo } \\
\text { r p }\end{array}$ & $\begin{array}{c}\text { Media } \\
\text { (IC95\%) }\end{array}$ & $\begin{array}{l}\text { Diferencia } \\
\text { (IC95\%) }\end{array}$ & $\begin{array}{l}\text { Valo } \\
\text { r p }\end{array}$ & $\begin{array}{c}\text { Media } \\
\text { (IC95\%) }\end{array}$ & $\begin{array}{l}\text { Diferencia } \\
\text { (IC95\%) }\end{array}$ & $\begin{array}{l}\text { Valo } \\
\text { r p }\end{array}$ \\
\hline $\begin{array}{l}\text { Función } \\
\text { física } \\
\text { Línea de } \\
\text { base }\end{array}$ & $\begin{array}{c}78,9 \\
(69,4 ; 88,5)\end{array}$ & --- & NA & $\begin{array}{c}83,4 \\
(73,9 ; 93,0)\end{array}$ & --- & NA & $\begin{array}{c}79,8 \\
(70,5 ; 89,0)\end{array}$ & --- & NA \\
\hline $\begin{array}{l}\text { Post- } \\
\text { intervenció } \\
n\end{array}$ & $\begin{array}{c}83,4 \\
(73,9 ; 93,0)\end{array}$ & $\begin{array}{c}4,5(- \\
3,5 ; 12,4)\end{array}$ & $\begin{array}{c}0,27 \\
1\end{array}$ & $\begin{array}{c}78,5 \\
(68,5 ; 88,5)\end{array}$ & $-5(-13,5 ; 3,6)$ & $\begin{array}{c}0,25 \\
5\end{array}$ & $\begin{array}{c}79,6 \\
(69,2 ; 90,0)\end{array}$ & $\begin{array}{c}-0,1(- \\
9,2 ; 8,9)\end{array}$ & $\begin{array}{c}0,97 \\
8\end{array}$ \\
\hline $\begin{array}{l}\text { Seguimient } \\
\mathrm{o}\end{array}$ & $\begin{array}{c}80,3 \\
(70,7 ; 89,8) \\
\end{array}$ & $1,3(-6,7 ; 9,3)$ & $\begin{array}{c}0,74 \\
6 \\
\end{array}$ & $\begin{array}{c}77,2 \\
(67,2 ; 87,2) \\
\end{array}$ & $\begin{array}{c}-6,2(- \\
14,8 ; 2,3)\end{array}$ & $\begin{array}{c}0,15 \\
1 \\
\end{array}$ & $\begin{array}{c}81,8 \\
(70,7 ; 93,0) \\
\end{array}$ & $\begin{array}{c}2,1(- \\
7,8 ; 12,0) \\
\end{array}$ & $\begin{array}{c}0,68 \\
2 \\
\end{array}$ \\
\hline Dolor & & & & & & & & & \\
\hline $\begin{array}{l}\text { Línea de } \\
\text { base }\end{array}$ & $\begin{array}{c}46,1 \\
(34,8 ; 57,3)\end{array}$ & --- & NA & $\begin{array}{c}52,3 \\
(41,1 ; 63,5)\end{array}$ & --- & NA & $\begin{array}{c}61,6 \\
(50,6 ; 72,5)\end{array}$ & --- & NA \\
\hline $\begin{array}{l}\text { Post- } \\
\text { intervenció } \\
n\end{array}$ & $\begin{array}{c}68,5 \\
(57,3 ; 79,7)\end{array}$ & $\begin{array}{c}22,5 \\
(10,7 ; 34,3)\end{array}$ & $\begin{array}{c}<0,0 \\
01\end{array}$ & $\begin{array}{c}51 \\
(39,0 ; 63,0)\end{array}$ & $\begin{array}{c}-1,3(- \\
13,9 ; 11,2)\end{array}$ & $\begin{array}{c}0,83 \\
4\end{array}$ & $\begin{array}{c}66,3 \\
(53,6 ; 79,0)\end{array}$ & $\begin{array}{c}4,7(- \\
8,4 ; 17,9)\end{array}$ & $\begin{array}{c}0,47 \\
9\end{array}$ \\
\hline $\begin{array}{l}\text { Seguimient } \\
\mathrm{o}\end{array}$ & $\begin{array}{c}65,9 \\
(54,7 ; 77,1) \\
\end{array}$ & $\begin{array}{c}19,8 \\
(8,1 ; 31,6) \\
\end{array}$ & $\begin{array}{c}0,00 \\
1 \\
\end{array}$ & $\begin{array}{c}54,2 \\
(42,2 ; 66,2) \\
\end{array}$ & $\begin{array}{c}1,9(- \\
10,6 ; 14,4) \\
\end{array}$ & $\begin{array}{c}0,76 \\
9 \\
\end{array}$ & $\begin{array}{c}63,2 \\
(49,3 ; 77,2) \\
\end{array}$ & $\begin{array}{c}1,7(- \\
12,7 ; 16,1)\end{array}$ & $\begin{array}{c}0,81 \\
7 \\
\end{array}$ \\
\hline $\begin{array}{l}\text { Salud } \\
\text { general } \\
\text { Línea de } \\
\text { base }\end{array}$ & $\begin{array}{c}49,1 \\
(39,8 ; 58,3)\end{array}$ & --- & NA & $\begin{array}{c}58,3 \\
(49,1 ; 67,6)\end{array}$ & --- & NA & $\begin{array}{c}57,2 \\
(48,2 ; 66,2)\end{array}$ & --- & NA \\
\hline $\begin{array}{l}\text { Post- } \\
\text { intervenció } \\
n\end{array}$ & $\begin{array}{c}71,8 \\
(62,5 ; 81,0)\end{array}$ & $\begin{array}{c}22,7 \\
(12,6 ; 32,9)\end{array}$ & $\begin{array}{c}<0,0 \\
01\end{array}$ & $\begin{array}{c}56,1 \\
(46,2 ; 66,1)\end{array}$ & $\begin{array}{c}-2,2(- \\
12,9 ; 8,6)\end{array}$ & $\begin{array}{c}0,69 \\
1\end{array}$ & $\begin{array}{c}58 \\
(47,5 ; 68,5)\end{array}$ & $\begin{array}{c}0,8(- \\
10,5 ; 12,1)\end{array}$ & 0,89 \\
\hline $\begin{array}{l}\text { Seguimient } \\
\mathrm{o}\end{array}$ & $\begin{array}{c}69,7 \\
(60,5 ; 79,0) \\
\end{array}$ & $\begin{array}{c}20,7 \\
(10,6 ; 30,8) \\
\end{array}$ & $\begin{array}{c}<0,0 \\
01\end{array}$ & $\begin{array}{c}56,2 \\
(46,3 ; 66,2) \\
\end{array}$ & $\begin{array}{c}-2,1(- \\
12,8 ; 8,7) \\
\end{array}$ & $\begin{array}{c}0,70 \\
3 \\
\end{array}$ & $\begin{array}{c}57,7 \\
(46,1 ; 69,3) \\
\end{array}$ & $\begin{array}{c}0,5(- \\
11,8 ; 12,8) \\
\end{array}$ & $\begin{array}{c}0,93 \\
8 \\
\end{array}$ \\
\hline Vitalidad & & & & & & & & & \\
\hline $\begin{array}{l}\text { Línea de } \\
\text { base }\end{array}$ & $\begin{array}{c}41,6 \\
(32,3 ; 50,8)\end{array}$ & --- & NA & $\begin{array}{c}52,1 \\
(42,8 ; 61,4)\end{array}$ & --- & NA & $\begin{array}{c}60,3 \\
(51,2 ; 69,3)\end{array}$ & --- & NA \\
\hline $\begin{array}{l}\text { Post- } \\
\text { intervenció } \\
n\end{array}$ & $\begin{array}{c}66,8 \\
(57,6 ; 76,1)\end{array}$ & $\begin{array}{c}25,3 \\
(15,7 ; 34,8)\end{array}$ & $\begin{array}{c}<0,0 \\
01\end{array}$ & $\begin{array}{c}51,5 \\
(41,6 ; 61,3)\end{array}$ & $\begin{array}{c}-0,7(- \\
10,8 ; 9,5)\end{array}$ & 0,9 & $\begin{array}{c}65,5 \\
(55,1 ; 75,9)\end{array}$ & $\begin{array}{c}5,2(- \\
5,4 ; 15,9)\end{array}$ & $\begin{array}{c}0,33 \\
6\end{array}$ \\
\hline $\begin{array}{l}\text { Seguimient } \\
\mathrm{o}\end{array}$ & $\begin{array}{c}64,7 \\
(55,5 ; 74,0) \\
\end{array}$ & $\begin{array}{c}23,2 \\
(13,6 ; 32,7) \\
\end{array}$ & $\begin{array}{c}<0,0 \\
01 \\
\end{array}$ & $\begin{array}{c}52,5 \\
(42,6 ; 62,4) \\
\end{array}$ & $\begin{array}{c}0,4(- \\
9,7 ; 10,6) \\
\end{array}$ & $\begin{array}{c}0,93 \\
5 \\
\end{array}$ & $\begin{array}{c}62,3 \\
(50,9 ; 73,7) \\
\end{array}$ & $\begin{array}{c}2,1(- \\
9,6 ; 13,7) \\
\end{array}$ & 0,73 \\
\hline $\begin{array}{l}\text { Función } \\
\text { social } \\
\text { Línea de } \\
\text { base }\end{array}$ & $\begin{array}{c}42,8 \\
(30,4 ; 55,1)\end{array}$ & --- & NA & $\begin{array}{c}59,9 \\
(47,5 ; 72,2)\end{array}$ & --- & NA & $\begin{array}{c}65 \\
(53,0 ; 77,0)\end{array}$ & --- & NA \\
\hline $\begin{array}{l}\text { Post- } \\
\text { intervenció } \\
n\end{array}$ & $\begin{array}{c}77 \\
(64,6 ; 89,3)\end{array}$ & $\begin{array}{c}34,2 \\
(21,0 ; 47,4)\end{array}$ & $\begin{array}{c}<0,0 \\
01\end{array}$ & $\begin{array}{c}61,5 \\
(48,3 ; 74,8)\end{array}$ & $\begin{array}{c}1,7(- \\
12,4 ; 15,7)\end{array}$ & $\begin{array}{c}0,81 \\
7\end{array}$ & $\begin{array}{c}70,2 \\
(56,2 ; 84,2)\end{array}$ & $\begin{array}{c}5,2(- \\
9,5 ; 19,9)\end{array}$ & $\begin{array}{c}0,48 \\
8\end{array}$ \\
\hline $\begin{array}{l}\text { Seguimient } \\
\mathrm{o}\end{array}$ & $\begin{array}{c}71,7 \\
(59,4 ; 84,1) \\
\end{array}$ & $\begin{array}{c}28,9 \\
(15,7 ; 42,2) \\
\end{array}$ & $\begin{array}{c}<0,0 \\
01 \\
\end{array}$ & $\begin{array}{c}56,1 \\
(42,9 ; 69,3) \\
\end{array}$ & $\begin{array}{c}-3,8(- \\
17,8 ; 10,3)\end{array}$ & 0,6 & $\begin{array}{c}74,4 \\
(59,0 ; 89,9) \\
\end{array}$ & $\begin{array}{c}9,4(- \\
6,6 ; 25,5) \\
\end{array}$ & 0,25 \\
\hline $\begin{array}{l}\text { Rol } \\
\text { emocional } \\
\text { Línea de } \\
\text { base }\end{array}$ & $\begin{array}{c}28,1 \\
(8,7 ; 47,4)\end{array}$ & --- & NA & $\begin{array}{c}45,6 \\
(26,3 ; 65,0)\end{array}$ & --- & NA & $\begin{array}{c}51,7 \\
(32,8 ; 70,5)\end{array}$ & --- & NA \\
\hline $\begin{array}{l}\text { Post- } \\
\text { intervenció } \\
n\end{array}$ & $\begin{array}{c}63,2 \\
(43,8 ; 82,5)\end{array}$ & $\begin{array}{c}35,1 \\
(12,3 ; 57,9)\end{array}$ & $\begin{array}{c}0,00 \\
3\end{array}$ & $\begin{array}{c}64,7 \\
(43,8 ; 85,6)\end{array}$ & $\begin{array}{c}19,1(- \\
5,0 ; 43,2)\end{array}$ & 0,12 & $\begin{array}{c}64,1 \\
(41,8 ; 86,3)\end{array}$ & $\begin{array}{c}12,4(- \\
12,7 ; 37,5)\end{array}$ & $\begin{array}{c}0,33 \\
3\end{array}$ \\
\hline $\begin{array}{l}\text { Seguimient } \\
\mathrm{o}\end{array}$ & $\begin{array}{c}68,4 \\
(49,1 ; 87,8)\end{array}$ & $\begin{array}{c}40,4 \\
(17,6 ; 63,1)\end{array}$ & $\begin{array}{c}0,00 \\
1\end{array}$ & $\begin{array}{c}52,5 \\
(32,6 ; 74,4)\end{array}$ & $\begin{array}{c}7,9(- \\
16,2 ; 32,0)\end{array}$ & $\begin{array}{c}0,51 \\
9\end{array}$ & $\begin{array}{c}69,8 \\
(45,1 ; 94,6)\end{array}$ & $\begin{array}{c}18,2(- \\
9,2 ; 45,5)\end{array}$ & $\begin{array}{c}0,19 \\
3\end{array}$ \\
\hline
\end{tabular}


Calidad de vida en cuidadores informales de pacientes con Alzheimer...

$\begin{array}{lccccccccc}\text { Línea de } & 45,1 & & & 63,6 & & & 67,8 & \text { NA } & \\ \text { base } & (36,9 ; 53,2) & --- & \text { NA } & (55,4 ; 71,7) & -- & & & (59,9 ; 75,7) & \text { NA } \\ \text { Post- } & 73,1 & 28 & <0,0 & 58,9 & -4,7(- & & 73,5 & 5,7(- & 0,23 \\ \text { intervenció } & (64,9 ; 81,2) & (19,5 ; 36,5) & 01 & (50,2 ; 67,6) & 13,7 ; 4,4) & 0,31 & (64,3 ; 82,7) & 3,8 ; 15,2) & 8 \\ \text { n } & 70,9 & 25,9 & <0,0 & 62,4 & -1,2(- & 0,79 & 79,2 & 11,4 & 0,03 \\ \text { Seguimient } & (62,8 ; 79,1) & (17,4 ; 34,4) & 01 & (53,7 ; 71,1) & 10,2 ; 7,9) & 6 & (69,1 ; 89,3) & (1,0 ; 21,7) & 2 \\ \text { o } & & & & & & & & & \end{array}$

Nota: Autoría propia.

En la Figura 1, se presentan los puntajes medios para el ítem de transición de la salud que pregunta sobre el cambio en el estado de salud general respecto al año anterior que va de 1 (mucho peor) a 5 (mucho mejor). Se encuentra que, el grupo multicomponente más respiro presenta una disminución de la media en 1,1 puntos, $\mathrm{p}<0,001$ en la percepción de su estado de salud comparado con el año previo, luego de la intervención tanto a los 5 como a los 10 meses de seguimiento.

En la Figura 2, se puede observar que, al analizar la calidad de vida por componentes físico y mental, se encontró que los cuidadores participantes de la intervención multicomponente más respiro percibieron un incremento de la calidad de vida en 4.4 puntos (IC 95\% $0,18 ; 8,7)$ posterior a la intervención $(\mathrm{p}=$ 0,041 ), mientras que en los demás grupos no se evidenciaron cambios significativos.

Por otro lado, en el componente mental, se evidenció un mayor efecto de la intervención multicomponente más respiro, aumentando la percepción de calidad de vida en 16,5 puntos (IC95\% $10,9 ; 22,1)$ de forma significativa $(\mathrm{p}<0,001)$ sosteniéndose el efecto incluso a 10 meses de seguimiento.

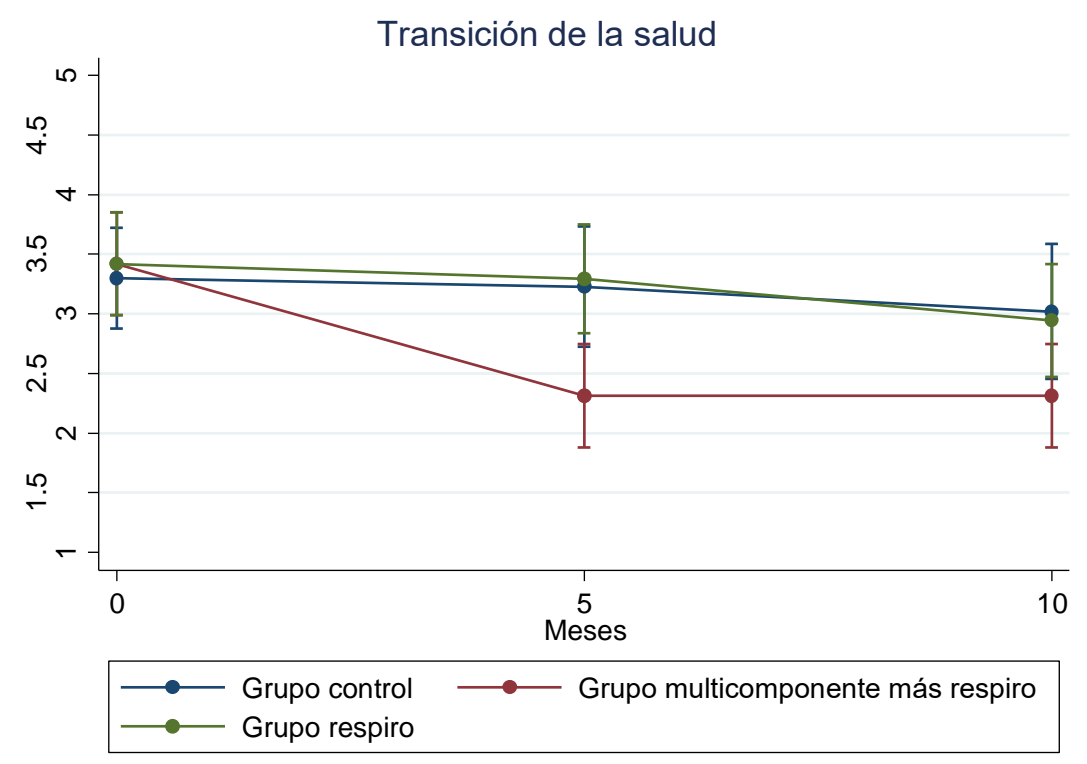

Figura 1. Puntaje de transición de la salud (SF-36) ajustados por modelo de efectos mixtos y diferencias según grupo de intervención y control durante el seguimiento. 


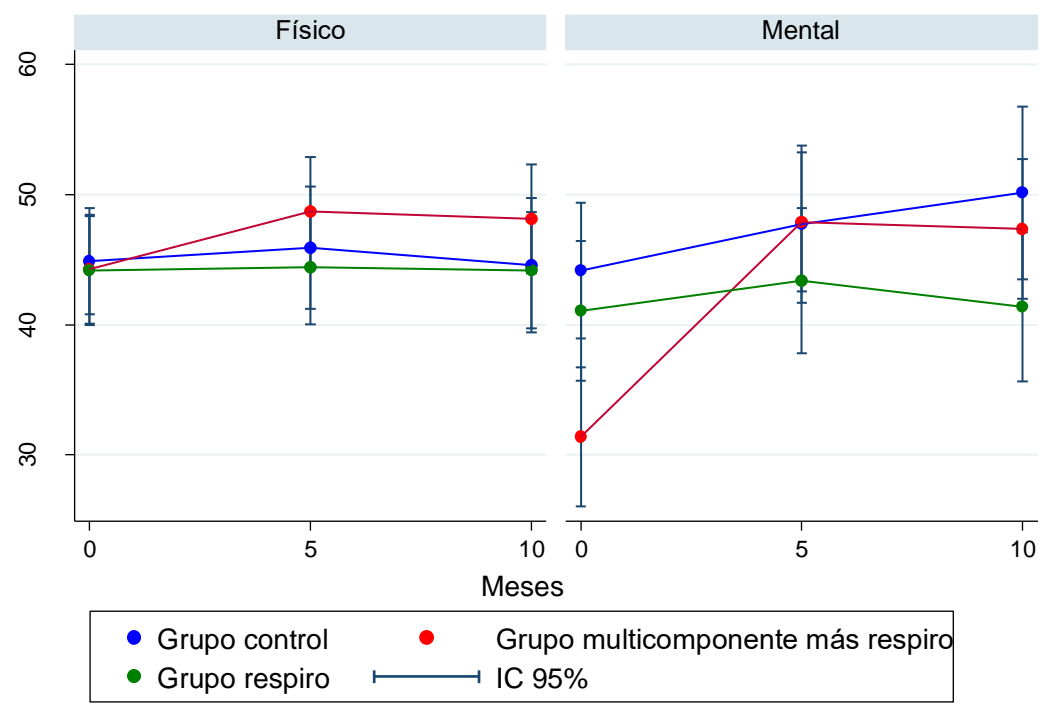

Figura 2. Puntaje medio de los componentes físico y mental de la calidad de vida (SF-36) ajustados por modelo de efectos mixtos y diferencias según grupo de intervención y control durante el seguimiento.

\section{DISCUSIÓN}

El estudio de los programas de intervención para cuidadores informales de pacientes con Alzheimer toma relevancia en la medida que sea viable la evaluación del impacto generado en la población, a partir de resultados que mejoren la calidad de vida de los cuidadores. La identificación de factores sociodemográficos en el perfil del cuidador informal puede otorgar claridad en las características de la población que se encuentra en mayor riesgo de sufrir las consecuencias del cuidado, para así generar medidas que busquen promover la salud desde un nivel primario de atención, así como la orientación para la priorización de la población que requiere intervención que en últimas se puede ver reflejado en un mejoramiento tanto de la atención brindada al paciente con Enfer- medad de Alzheimer como del cuidado del cuidador informal que se encuentra en riesgo de convertirse en un paciente oculto.

De la población evaluada, el grupo que se presenta con mayor frecuencia en el perfil del cuidador informal del paciente con Enfermedad de Alzheimer es el género femenino; características que se asemejan a los resultados presentes en Kasper, Freedman, Spillman y $\mathrm{Wolff}^{24}$ y Yu, Wang, He, Liang y Zhou ${ }^{25}$ aunque esta predominancia de género también se conserva en la población Colombiana ${ }^{26}$. Lo anterior, destaca la realidad de cómo la población femenina, por el hecho de ser cuidadora, se enfrenta a un mayor riesgo padecer algún tipo de patología derivada del oficio del cuidado, así como una posible disminución de la calidad de vida, como parte del fenómeno de feminización del cuidado. 
Calidad de vida en cuidadores informales de pacientes con Alzheimer...

Por otra parte, se afirma que el perfil altruista de los cuidadores informales, el cual genera una tendencia a buscar el bien de las personas de forma desinteresada, los hace pensarse como merecedores de un menor cuidado y atención, generando descuidos en su propia salud, que llevan a mayores comorbilidades a mediano y largo plazo y que por consiguiente alteran su bienestar y calidad de vida. Por otra parte, se ha planteado la necesidad de comprender con mayor profundidad las líneas de acción de las personas involucradas (en este caso cuidadores informales), tales como la forma del cuidado, el manejo del tiempo y la apropiación de su labor, así como del entendimiento de la razón u objetivo que busca el cuidador informal y así mismo, el sentido de vida que se proyecta, al estar relacionado con un familiar que presenta una enfermedad neurodegenerativa crónica como lo es el Alzheimer ${ }^{6}$. En este sentido, es de resaltar que el presente programa multicomponente permitió a los cuidadores informales, obtener un rendimiento mayor y más eficiente en el tiempo que dedican al desarrollo de sus actividades diarias, con menor interferencia de problemas emocionales, lo cual se determina por las mejorías en el rol emocional, en su salud física y mental y en su calidad de vida.

Se ha reportado la existencia de una fuerte correlación entre percepción de la calidad de vida del cuidador y la de los pacientes, y de manera sorprendente, se encontró una mejor puntuación de estos últimos, esta correlación se vio afectada por la edad del cuidador, la edad al inicio de los síntomas del paciente, el deterioro de las actividades de la vida diaria de los pacientes, el tiempo dedicado al cuidado y los síntomas depresivos. También, la calidad de vida del cuidador fue más baja cuando mayor era el deterioro del paciente $^{27}$. En el presente análisis, los puntajes de calidad de vida de los cuidadores estuvieron en promedio entre los 40 y 50 puntos para el componente físico y entre 25 y 50 puntos en el componente mental, los cuales se encuentran por debajo a lo percibido por los cuidadores de pacientes con alteraciones musculoesqueléticas, como la osteoartritis y fibromialgias ${ }^{28}$.

En relación con las implicaciones físicas de la labor del cuidado, algo semejante ocurre en el desarrollo del bienestar físico del cuidador de familiares con enfermedades neurodegenerativas como Alzheimer, las cuales son presentadas como condiciones de salud de tipo no transmisibles y afecciones musculoesqueléticas, derivadas de comportamientos sedentarios y posturas inadecuadas por dar prioridad al cuidado de su familiar ${ }^{29}$. Estas similitudes se encuentran en estudios donde se evalúa la sobrecarga psicofísica en familiares cuidadores de pacientes inmovilizados $^{30}$. Dicha sobrecarga psicofísica, en este estudio, tras la implementación del programa multicomponente, mejoró, lo cual se puede entender desde la dimensión de dolor corporal, traduciéndose en la intensidad de dolor que el cuidador siente en la realización de sus actividades diarias, siendo evidentes los resultados en esta dimensión, sin embargo, sus efectos no se mantienen en el tiempo, con lo cual se deduce que la educación postural permite al cuidador entender su autocuidado, pero requiere de un trabajo permanente en el tiempo, para que se apropie conscientemente de ello. 
Cabe destacar que la intervención de programas multicomponentes en salud para cuidadores informales de personas con Enfermedad de Alzheimer, han reportado impactos favorables a corto plazo que se mantienen en el tiempo en áreas del desarrollo personal, tales como área psicológica, física y social; a su vez permite al cuidador informal el empoderamiento en el conocimiento de áreas de su salud sobre el cuidado de su más próximo ${ }^{31}$.

Al mismo tiempo, este estudio presenta cambios positivos en las variables de autopercepción, calidad de vida y función física, sin embargo, aunque esta última no presenta diferencias significativas, aun así los resultados en la función física de este estudio dan evidencia de la necesidad de un programa que incluya intervenciones que puedan fortalecer esta dimensión, en parte por los altos porcentajes de alteraciones reportados en la dimensión física, especialmente de tipo musculoesquelético, que son evidentes durante los momentos de cuidado básico, como actividades de aseo personal para el paciente, puesto que involucran movilizaciones corporales con gran carga muscular por actividades de carga, descarga, empuje $\mathrm{o}$ arrastre de peso ${ }^{32}$, entre otros aspectos.

$\mathrm{Al}$ igual que en el presente análisis, la intervención de componentes múltiples ha mostrado efectos significativos en el mejoramiento de la calidad de vida basados en la práctica del cuidado ${ }^{33}$, así mismo, en un meta-análisis se encontró que las intervenciones multicomponente tienen un efecto positivo y a pesar de que el tamaño del efecto fue pequeño, recomiendan este tipo de intervenciones por considerarse de bajo costo y de amplia aceptabilidad $^{34}$. En el estudio se observó que el grupo multicomponente más respiro presenta un cambio positivo en la percepción de si estado de salud comparado con el año previo, luego de la intervención tanto a los 5 como a los 10 meses de seguimiento (disminución de la media en 1,1 puntos, $\mathrm{p}<0,001$ ), mientras que, para el grupo de respiro y control, no hubo cambios significativos en la percepción del estado de salud. En contraparte, Tanner y colaboradores, no reportaron diferencias significativas en la calidad de vida para una intervención multicomponente de base comunitaria $(\mathrm{p}=0,257)^{35}$.

Para finalizar, es por ello que se hace necesario reforzar la idea que se presenta en estudios en el ámbito mundial donde determinan el impacto favorable que resulta del desarrollo de un programa de intervención psicosocial multicomponente, que consiste en intervenciones desde psicoeducación y componentes cognitivos donde los cuidadores informales son los participantes principales ${ }^{17-}$ ${ }^{36}$, así mismo, se refuerza la importancia de incluir dichos programas en los servicios integrales que se le brindan a las personas con trastornos neurocognitivos ${ }^{31}$. Este estudio involucró, dentro del programa multicomponente, secciones de educación en higiene postural básica, movilización del paciente, ejercicios físicos al despertar, adecuada postura al dormir, entre otros, que aportaron al cuidador el conocimiento primario para adoptar posturas que minimizaran su riesgo musculoesquelético, resultados consecuentes con las puntuaciones en función física obtenidas tras la implementación del programa multicomponente, siendo este programa un punto inicial para que cada cuidador 
Calidad de vida en cuidadores informales de pacientes con Alzheimer...

informal, personalice y entienda, lo que pasa en su vida y en la del paciente con Enfermedad de Alzheimer que cuida, comprendiendo que el Alzheimer es un trastorno degenerativo, por ende, con el transcurso de los años, la labor de cuidado se hace más ardua, implicando mayor trabajo físico y psicológico.

Sin duda alguna, las intervenciones no farmacológicas presentan mejoras estadísticamente significativas sobre la calidad de vida de los cuidadores informales, evidenciado en este estudio con la implementación del programa multicomponente y respiro, el cual fue desarrollado con enfoque y acciones psicoterapéuticas, educativas y multicomponentes, así como la presencia de grupos de apoyo, lo cual coincide con otros hallazgos del área ${ }^{18}$.

Dentro de las limitaciones encontradas en el desarrollo del presente estudio, se encuentran la disposición de los familiares cuidadores a aceptar la participación en los encuentros semanales, no por su motivación, sino por la demanda del cuidado del paciente, lo que disminuyó el potencial de la participación. Sin embargo, cabe resaltar que, una vez iniciado el programa, los participantes cumplieron con los objetivos del programa, además de reportar de manera cualitativa los efectos positivos en la labor del cuidado. A su vez, se recomienda reforzar el componente relacionado con la dimensión física en el programa multicomponente de manera que se logre un efecto balanceado entre lo físico y mental, dado que el programa obtuvo un mayor impacto en el aspecto psicológico de la calidad de vida de los cuidadores.
Como recomendación adicional, se considera la continuidad de programas de intervención donde se afiancen las características personales, se potencien las habilidades del cuidado y se refuercen acciones integrales que beneficien la dupla cuidador-paciente. Actualmente, en Colombia, no existen programas orientados a reducir la sobrecarga del cuidador, ni tampoco a mejorar su calidad de vida, y menos un reconocimiento económico por su labor. Otros países como España, han generado jurisprudencia en torno a la labor del cuidado con la Ley de Promoción de la Autonomía Personal y Atención a las personas en situación de dependencia, como una forma de reconocimiento al derecho a recibir atención digna, así como la prestación económica por cuidados en el entorno familiar y apoyo a cuidadores no profesionales, dignificando la labor del cuidado que brindan los familiares ${ }^{37}$.

Por lo tanto, impera que, desde la política pública, se den pasos para la creación de mecanismos que busquen sostener el recurso humano aportado por los cuidadores informales y las familias, a través de alianzas entre los sistemas de salud, las organizaciones y los servicios comunitarios, y con el apoyo de la formación que las universidades pueden brindar para ayudar a optimizar los cuidados que los familiares brindan.

Para el sistema de salud, la atención no solo del paciente de Alzheimer, sino de las necesidades y problemas de salud de los cuidadores, implica un desafío desde el ámbito preventivo, así como el ofrecimiento de posibilidades de intervención con la mejor evidencia disponible, para mejorar la calidad de vida de los cuidadores ${ }^{38}$. 
Cerquera Córdoba AM, Tiga Loza DC, Álvarez Anaya WA, Dugarte Peña E, Plata Osma LJ, Jaimes Espíndola LR

Por otro lado, pese a que en el presente estudio la intervención de respiro no mejoró los niveles de calidad de vida de los cuidadores, es una intervención que debe tenerse en cuenta para que programas como el multicomponente puedan operar, permitiendo la asistencia de los cuidadores a través del relevo. Es así como se requiere de esfuerzos tanto del estado como de la sociedad civil para el desarrollo y sostenimiento de programas que atiendan las necesidades de los cuidadores informales, y que se les reconozca como trabajadores pese a la informalidad de su tarea y el poco entrenamiento de este más allá de la experiencia vivida. Lo anterior se podría lograr de manera inicial con la creación de una ley que busque reconocer y atender al cuidador informal, y materializar su contenido con la instauración de programas sostenibles, aceptados por la comunidad y con impacto en la salud de las familias.

\section{CONCLUSIONES}

La implementación de un programa multicomponente en cuidadores informales de pacientes con TNC tipo Alzheimer, es efectivo en mejorar la calidad de vida y la percepción de autocuidado, con mejorías que perduran en el tiempo, lo que facilita la acción de cuidar y a largo plazo evitará comorbilidades en el cuidador informal.

El cuestionario SF36, mostró resultados satisfactorios en todas sus dimensiones, estos valores pueden utilizarse como valores de referencia que permitan monitorizar el impacto generado en el autocuidado de los cuidadores informales.

Finalmente, las intervenciones no farmacológicas, con énfasis en psicoe- ducación y autocuidado, en cuidadores informales, aportan beneficios significativos que promueven y personalizan su propio cuidado durante la labor de cuidar.

\section{AGRADECIMIENTOS}

Equipo de investigación del proyecto Evaluación de impacto de un programa multicomponente con intervención transdisciplinar en la sobrecarga y la calidad de vida relacionada a la salud de cuidadores informales de pacientes con Trastorno Neurocognitivo (TNC) mayor tipo Alzheimer en Bucaramanga y su área metropolitana. Proyecto de investigación financiado por el Ministerio de Ciencia, Tecnología e Innovación (MINCIENCIAS), convocatoria 807 -2018 para proyectos de ciencia, tecnología e innovación en salud.

\section{CONFLICTO DE INTERESES}

Los autores declaran no tener conflicto de intereses.

\section{REFERENCIAS BIBLIOGRÁFICAS}

1. Organización Mundial de la Salud. Enfermedades no transmisibles. 13 de abril de 2021.

2. Organización Mundial de la Salud. Demencia. 21 de septiembre de 2020.

3. Organización Panamericana de la Salud. Campaña antiestigma urge a hablar sobre demencia. 2020.

4. Ministerio de Salud y Protección Social, Colombia. Boletín de salud mental Demencia. 2017.

5. Zabala-Gualtero JM, Cadena-Sanabria MO. Enfermedad de Alzheimer y Síndrome de Carga del Cuidador: la importancia de cuidar al cuidador. Revista Médicas UIS. 2018;31(1):9-13. 
Calidad de vida en cuidadores informales de pacientes con Alzheimer...

6. Garre-Olmo J. Epidemiología de la enfermedad de Alzheimer y otras demencias. Revista de Neurología. 2018;66:377-86.

7. Montgomery, W., Goren, A., KahleWrobleski, K., Nakamura, T., \& Ueda, K. Alzheimer's disease severity and its association with patient and caregiver quality of life in Japan: Results of a community-based survey. BMC Geriatrics. 2018;18(1):1-12.

8. Galvis-López C, Aponte-Garzón L, Pinzón-Rocha M. Percepción de la calidad de vida de cuidadores de pacientes asistentes a un programa de crónicos, Villavicencio, Colombia. Aquichan. 2016;16(1):104-15.

9. Martín Á, Rivera J. Tendencias demográficas a escala mundial y sus repercusiones en la provisión de cuidado a las personas mayores dependientes. Actas de Coordinación Sociosanitaria. 2019;(25):31-58.

10. Liu W, Lyu T, Zhang X, Yuan S, Zhang $\mathrm{H}$. Willingness-to-pay and willingnessto-accept of informal caregivers of dependent elderly people in Shanghai, China. BMC Health Services Research [Internet]. 2020;20(618). Disponible en:

https://bmchealthservres.biomedcentral .com/articles/10.1186/s12913-02005481-2

11. Lumbreras AV, Orellán RG. Cuidado informal y feminización de la pobreza: retos y emergencias. Antropologías en transformación: sentidos, compromisos y utopías [Internet]. 2017;1073-80.

12. Naughton MJ, Shumaker SA, Anderson RT, Czajkowski SM. Psychological Aspects of HealthRelated Quality of Life Measurement: Tests and Scales.
Quality of Life and Pharmaco economics in Clinical Trials. 1996;15:117-31.

13. de María Cáceres-Manrique F, ParraPrada LM, Pico-Espinosa OJ. Calidad de vida relacionada con la salud en población general de Bucaramanga, Colombia. Revista de Salud Pública. 2018;20(2):147-54.

14. Robles-Espinoza AI, Rubio-Jurado B, De la Rosa-Galván EV, Nava-Zavala AH. Generalidades y conceptos de calidad de vida en relación con los cuidadores de salud. Residente. 2016;11(3):120-5.

15. Lugo LE, García HI, Gómez C. Confiabilidad del cuestionario de calidad de vida en salud SF-36 en Medellin, Colombia. Revista Facultad Nacional de Salud Pública. 2006;24(2):37-50.

16. Garzón-Maldonado FJ, GutiérrezBedmar M, García-Casares N, PérezErrázquin F, Gallardo-Tur A, MartínezValle Torres MD. Health-related quality of life in caregivers of patients with Alzheimer disease. Neurología (English Edition). 2017;32(8):508-15.

17. Abrahams R, Liu KPY, Bissett M, Fahey P, Cheung KSL, Bye R, et al. Effectiveness of interventions for coresiding family caregivers of people with dementia: Systematic review and meta-analysis. Australian Occupational Therapy Journal. 2018;65(3):208-24.

18. Amador-Marín B, Guerra-Martín MD. Eficacia de las intervenciones no farmacológicas en la calidad de vida de las personas cuidadoras de pacientes con enfermedad de Alzheimer. Gaceta. 2017;31(2):154-60. 
Cerquera Córdoba AM, Tiga Loza DC, Álvarez Anaya WA, Dugarte Peña E, Plata Osma LJ, Jaimes Espíndola LR

19. Cerquera A, Tiga D, Álvarez W, Plata L. Ensayo controlado aleatorizado de un programa multicomponente para cuidadores informales de pacientes con Alzheimer. 2020.

20. Ware J. SF-36 health survey update. Spine. 2000;25(24):3130-9.

21. Romero Massa E. Reliability of the SF36 questionnaire in post acute myocardial infarction patients from the city of Cartagena de Indias, Colombia. Revista Colombiana de Cardiologia,. 2010;17(2):41-6.

22. Lp. S. Stata Statistical Software: Release 16. Patent. 13-SE, 2019.

23. Consejo de Organizaciones Internacionales de las Ciencias Médicas (CIOMS) y Organización Mundial de la Salud (OMS). Pautas éticas internacionales para la investigación relacionada con la salud con seres humanos. Ginebra, 2016.

24. Kasper JD, Freedman VA, Spillman BC, Wolff JL. The Disproportionate Impact Of Dementia On Family And Unpaid Caregiving To Older Adults Judith. Health Aff (Millwood). 2015;34(10):1642-9.

25. Yu H, Wang X, He R, Liang R, Zhou L. Measuring the caregiver burden of caring for community-residing people with Alzheimer's disease. PLoS ONE. 2015;10(7):1-13.

26. Chaparro-Díaz L, Barrera-Ortiz L, Vargas-Rosero E, Carreño-Moreno SP. Mujeres cuidadoras familiares de personas con enfermedad crónica en Colombia. Revista Ciencias de la Salud. 2016;13(1):72-86.

27. Schumann C, Alexopoulos P, Perneczky R. Determinants of self- and carer-rated quality of life and caregiver burden in Alzheimer disease. International Journal of Geriatric Psychiatry. 2019;34(10):1378-85.

28. Iguti AM, Guimarães M, Barros MBA. Health-related quality of life (SF-36) in back pain: a population-based study, Campinas, Sao Paulo State, Brazil. Cadernos de Saúde Pública. 2021;37(2):1-12.

29. Acuña MR, Doren FM, Campos S, Rojas CA. Adaptando mi vida: vivencias de cuidadores familiares de personas con enfermedad de Alzheimer. Geroko. 2018;29(2):54-8.

30. Vérez N. Detección de sobrecarga y psicopatologías asociadas a cuidadores principales familiares de enfermos de Alzheimer en Galicia. [España]: Universidad Nacional de Educación a Distancia; 2016.

31. Shata ZN, Amin MR, El-Kady HM, Abu-Nazel MW. Efficacy of a multicomponent psychosocial intervention program for caregivers of persons living with neurocognitive disorders, Alexandria, Egypt: A randomized controlled trial. Avicenna Journal of Medicine. 2017;7(2):54-63.

32. Piñeiro I, Rodríguez S, Albite A, Freire C, del Mar Ferradás M. Sobrecarga y salud percibida en cuidadores informales de pacientes con enfermedad mental. European Journal of Health Research. 2017;3(3):185-96.

33. Nguyen TA, Nguyen $H$, Pham $T$, Nguyen TH, Hinton L. A cluser randomized controlled trial to test the feasibility and preliminary effectiveness of a family dementia caregiver intervetion in Vietnam. Medicine (Baltimore). 2018;97(42):15. 
Calidad de vida en cuidadores informales de pacientes con Alzheimer...

34. Laver K, Milte R, Dyer S, Crotty M. A Systematic Review and Meta-Analysis Comparing Carer Focused and Dyadic Multicomponent Interventions for Carers of People With Dementia. Journal of Aging and Health [Internet]. 2017;29(8). Disponible en: http://dx.doi.org/10.1177/0898264316 660414

35. Tanner JA, Black BS, Johnston D, Hess E, Leoutsakos J-M, Gitlin LN, et al. A randomized controlled trial of a community-based dementia care coordination intervention: Effects of MIND at home on caregiver outcomes. American Journal of Geriatric Psychiatry. 2015;23(4):391-402.

36. Huis In Het Veld J, Verkaik R, Mistiaen P, van Meijel B, Francke AL. The effectiveness of interventions in supporting self-management of informal caregivers of people with dementia; A systematic meta review Health services research. BMC Geriatrics. 2015;15(1):1-10.

37. Hu X, Dolansky MA, Su Y, Hu X, Qu M, Zhou L. Effect of a multidisciplinary supportive program for family caregivers of patients with heart failure on caregiver burden, quality of life, and depression: a randomized controlled study. International Journal of Nursing Studies. 2016; 62:11-21.

38. Fuhrmann AC, Bierhals CCB, Day CB, Mocellin D, de Medeiros GG, Dos Santos NO, et al. Experiencias y retos al cuidar de un familiar anciano con accidente cerebrovascular. revista cubana [Internet]. 2020;36(2). Disponible en: http://www.revenfermeria.sld.cu/index .php/enf/article/view/3173 